Obere Extremität 2020 $15: 160-171$ https://doi.org/10.1007/s11678-020-00585-x Eingegangen: 23. April 2020

Angenommen: 29. Juni 2020

Online publiziert: 31 . Juli 2020

(c) Springer Medizin Verlag GmbH, ein Teil von Springer Nature 2020

Jan-Philipp Imiolczyk ${ }^{1}$ Florian Freislederer ${ }^{2}$ Patric Raiss ${ }^{3} \cdot$ Markus Scheibel $^{1,2}(\mathbb{D}$

${ }^{1}$ Centrum für Muskuloskeletale Chirurgie (CMSC), Charité-Universitätsmedizin Berlin, Berlin, Deutschland ${ }^{2}$ Schulthess Klinik Zürich, Zürich, Schweiz

${ }^{3}$ Orthopädische Chirurgie München (OCM), München, Deutschland

\title{
Langzeitergebnisse in der inversen Schulterendoprothetik
}

erweitert, sodass gegenwärtig weltweit mehr inverse Endoprothesen im Vergleich zu anatomischen Endoprothesen eingesetzt werden [2].

Diese Übersichtsarbeit befasst sich mit den aktuell publizierten Langzeitergebnissen der iSTEP basierend auf dem oben genannten Konzept von Paul Grammont.

\section{Irreparable RM-MR bzw.} Defektarthropathie

Irreparable Rotatorenmanschetten-Massenrupturen (RM-MR) bzw. Defektarthropathien („cuff-tear arthropathy“, CTA) werden unterteilt in Pathologien ohne (Hamada- und Fukuda-Stadium 1-3) und mit begleitender Omarthrose (Hamada- und Fukuda-Stadium 4-5; [3]).

Patienten mit irreparablen Defekten der RM weisen früh, aufgrund der muskulären Dysbalance, eine superiore Migration des Humeruskopfes über eine zur Azetabularisierung bis hin $\mathrm{zu}$ einer Humeruskopfnekrose auf (- Abb. 1a, b). Dieses Patientenkollektiv ist jung $(<65$ Jahre) und zeigt häufig vermehrte Voroperationen auf, da Therapieversuche mittels Débridement, RMRekonstruktionen oder Sehnentransfers ausgeschöpft werden, bevor die iSTEP als Ultima Ratio verwendet wird.

Gonzales et al. [4] konnten 112 Patienten mit einer primären, nicht-voroperierten RM-MR (Hamada- und Fukuda-Stadium 1-3) nach 97 (60-246) Monaten nachuntersuchen. Der ConstantMurley-Score (CS) verbesserte sich signifikant von 28 (5-61) auf 61 (23-85) Punkte beiZunahme in allen Bewegungsumfängen (ROM; Flexion: 70 auf $137^{\circ}$;
Außenrotation: $14^{\circ}$ auf $19^{\circ}$ ) mit Ausnahme in Innenrotation. Die Patienten wurden abhängig von ihrem Nachuntersuchungszeitraum aufgeteilt: Patienten mit einer Nachuntersuchung (NU) von über 8 Jahren (42\%) zeigten eine signifikante Abnahme in den CS-Subkategorien Schmerz und Aktivitäten auf, die Tendenz der ROM war auch rück-

\begin{tabular}{|c|c|}
\hline \multicolumn{2}{|c|}{ Abkürzungen } \\
\hline$a C S$ & $\begin{array}{l}\text { Alters- und geschlechtsadaptierter } \\
\text { Constant-Murley-Score }\end{array}$ \\
\hline ASES & $\begin{array}{l}\text { American Shoulder and Elbow } \\
\text { Surgeons Shoulder Score }\end{array}$ \\
\hline CS & Constant-Murley-Score \\
\hline CTA & Defektarthropathie \\
\hline FFX & Frakturfolgezustände \\
\hline HEP & Hemiendoprothese \\
\hline$H K F$ & Proximale Humeruskopffrakturen \\
\hline iSTEP & $\begin{array}{l}\text { Inverse Schultertotalendoprothe- } \\
\text { se }\end{array}$ \\
\hline NU & Nachuntersuchung \\
\hline$O A$ & Primäre Omarthrose \\
\hline ORIF & Plattenosteosynthese \\
\hline$R A$ & Rheumatoide Arthrits \\
\hline$R M$ & Rotatorenmanschette \\
\hline$R M-M R$ & $\begin{array}{l}\text { Irreparable Rotatorenmanschet- } \\
\text { ten-Massenrupturen }\end{array}$ \\
\hline ROM & $\begin{array}{l}\text { Bewegungsumfang („range of } \\
\text { motion“) }\end{array}$ \\
\hline$S N$ & Skapuläres Notching \\
\hline SSV & „Subjective shoulder value" \\
\hline TEP & $\begin{array}{l}\text { Anatomische Schultertotalendo- } \\
\text { prothese }\end{array}$ \\
\hline
\end{tabular}



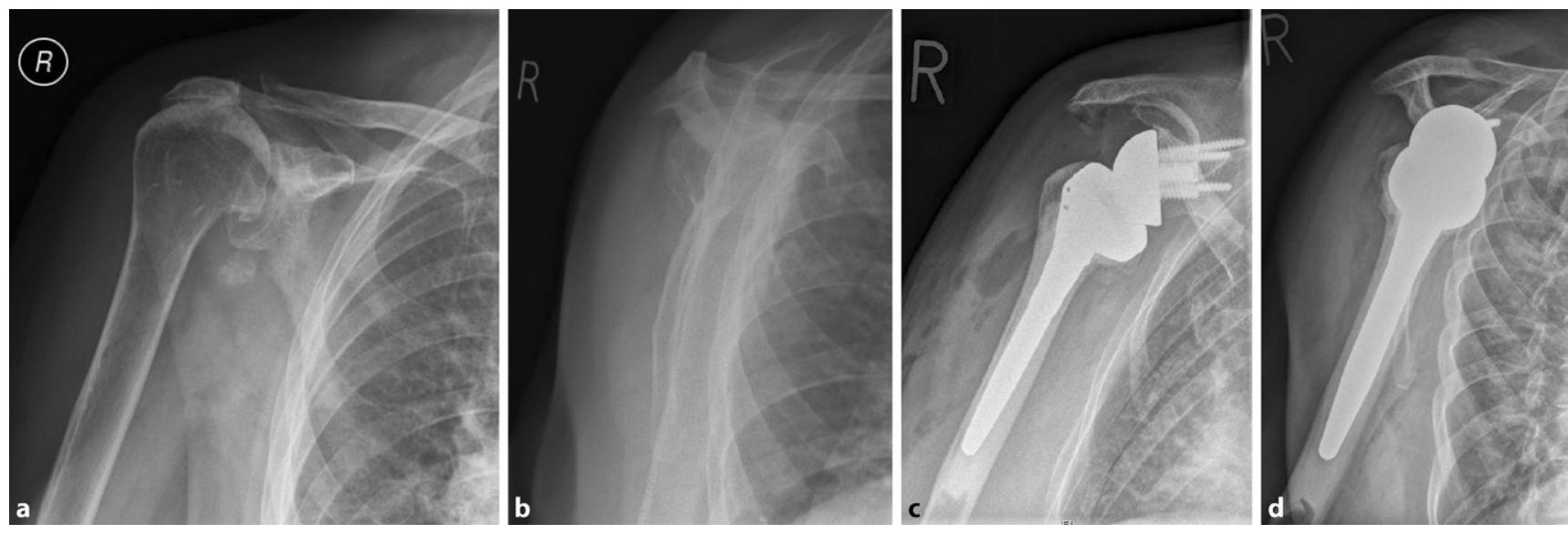

Abb. $1 \Delta$ a-d (von links nach rechts): Versorgung einer Defektarthropathie mittels „Grammont-iSTEP“ (inverse Schultertotalendoprothese). Röntgen einer ausgeprägten Defektarthropathie mit superiorer Migration und Azetabularisierung des Humeruskopfes in True-a.-p.- (a) und axialer (b) Aufnahme. Versorgung mittels iSTEP nach Paul Grammont mit $155^{\circ}{ }_{\text {"neck-shaft }}$ angle" in True-a.-p.- (c) und axialer (d) Röntgenaufnahme

läufig, wenn auch nicht statistisch signifikant. Des Weiteren schlussfolgerten die Autoren, dass die präoperative Funktion einen Einfluss auf das finale funktionelle Ergebnis hat: Patienten mit Schultersteife erzielen weniger ROM (Flexion: $111^{\circ}$ vs. $142^{\circ}$; Außenrotation: $11^{\circ}$ vs. $21^{\circ}$ ) und weniger Punkte im CS (53 vs. 63) als Patienten mit einer präoperativen, passiven globalen Abduktion von $>90^{\circ}$.

Ek et al. [5] legen ihr Augenmerk auf das junge Patientenkollektiv unter 65 Jahren, Ernstbrunner et al. [6] befassen sich gar ausschließlich mit unter 60-Jährigen. Die Delta III-Endoprothese (DePuy Synthes Inc., Warsaw, Indiana, USA) fand in $80 \%$ [5] bzw. $61 \%$ [6] Verwendung; die restlichen Patienten wurden mit der Anatomical Shoulder Reverse (Zimmer Biomet Holdings Inc., Warsaw, Indiana, USA) versorgt.

Ek et al. [5] konnten 40 Patienten (46-64 Jahre) mit einer durchschnittlichen NU von knapp 8 Jahren einschließen, von denen $58 \%$ mindestens eine Voroperation aufwiesen. Bei einer Komplikationsrate von $37,5 \%$ mussten $15 \%$ revidiert werden; $21 \%$ wiesen bei der finalen NU ein skapuläres Notching (SN) von Grad 3-4 auf. Abgesehen von der Kraft $(4,1$ vs. $5,0 \mathrm{~kg})$ weisen Patienten mit Voroperationen bessere funktionelle und subjektive Ergebnisse auf (CS: 60 vs. 53 Punkte; "subjective shoulder value" [SSV]: $68 \%$ vs. $61 \%$; Flexion: $126^{\circ}$ vs. $\left.109^{\circ}\right)$.
Ernstbrunner et al. [6] beschreiben gute klinische Ergebnisse (CS: $59 \pm 19$ Punkte; Flexion: $117 \pm 34^{\circ}$; SSV: $71 \pm 27 \%$ ) bei $95 \%$ SN nach über 10 Jahren, wobei im Vergleich zu den NUErgebnissen von 8-10 Jahren ein Leistungsknick von 5 Punkten im CS und $7 \% \mathrm{im} \mathrm{SSV} \mathrm{zu} \mathrm{verzeichnen} \mathrm{ist.} \mathrm{Insgesamt}$ waren $65 \%$ der Patienten voroperiert. Es wurden Komplikationen von 39\% und ein SN Grad 3-4 in 29\% der Fälle beobachtet. $26 \%$ mussten aufgrund von Infektionen oder Glenoidlockerungen revidiert werden und ähnlich den Revisionen bei Ek et al. [5] wurde eine Explantation und Konversion zur Hemiendoprothese (HEP) angestrebt [6].

Gerber et al. [7] verweisen trotz einer hohen Rate an Komplikationen (59\%) und daraus resultierenden Reoperationen (55\%) nach über 15 Jahren auf gute klinische Ergebnisse (CS: $58 \pm 19$ Punkte; Flexion: $101 \pm 27^{\circ}$; SSV: $78 \pm 26 \%$ ) und eine hohe Patientenzufriedenheit $(63 \%$ exzellent) sofern, die Explantation der iSTEP verhindert werden konnte. 6 von 22 eingeschlossenen Patienten (27\%) mussten aufgrund Versagens auf eine HEP konvertiert werden und wurden aus den klinischen Ergebnissen ausgeschlossen.

Cuff et al. [8] und Bacle et al. [9] veröffentlichten 2017 zwei Langzeitstudien, die heterogene Patientengruppen zusammenfassen und diese Subgruppen differenziert analysieren.
Bacle et al. ([8]; CTA; $n=27$ ) beobachteten eine Verbesserung des (CS) von präoperativ $22( \pm 11)$ Punkten auf mittelfristig $70( \pm 11)$ und letztlich auf 63 $( \pm 13)$ nach über 10 Jahren. Obwohl sich der Bewegungsumfang nicht veränderte ( $29 \pm 6$ Punkte), ließ die Abduktionskraft im Vergleich zu mittelfristigen NU bei relativ stabiler Flexion $\left(147^{\circ} \pm 17\right.$ auf $\left.146^{\circ} \pm 26\right)$ im Schnitt um fast $2 \mathrm{~kg}$ nach.

Cuff et al. ([9]; CTA: $n=19$ ) verzeichneten eine Verbesserung des American Shoulder and Elbow Surgeons Shoulder Score (ASES) von 36 (0-63) Punkten auf 79 (17-100) nach 5 Jahren und bis hin zu 76 (58-90) Punkten nach 10 Jahren. Bei einer Abduktion von $137^{\circ}$ (45-180) und aktiver Außenrotation von $53^{\circ}(0-90)$ lag die durchschnittliche Flexion bei $132^{\circ}(10-180)$. Im Vergleich von 5- zu 10-Jahres-NU wurden keine signifikanten Bewegungsverschlechterungen festgestellt (alle Ebenen), wobei $70 \%$ der Patienten keine Schmerzprogredienz oder eine weitere Schmerzreduktion beschrieben.

Bassens et al. [10] untersuchten $58 \mathrm{~Pa}$ tienten mit CTA nach primärer Versorgung mit Delta Xtend (DePuy Synthes Inc., Warsaw, Indiana, USA) nach. Bei einer durchschnittlichen NU von fast 10 Jahren wurde ein alters- und geschlechtsadaptierter CS (aCS) von 81 $( \pm 19)$ Punkten erreicht [10].

Trotz guter funktioneller Ergebnisse nach 10 Jahren lässt sich zwischen mittel- und langfristigen Ergebnissen 
Obere Extremität 2020 • 15:160-171 https://doi.org/10.1007/s11678-020-00585-x

(c) Springer Medizin Verlag GmbH, ein Teil von Springer Nature 2020

\section{J.-P. Imiolczyk · F. Freislederer · P. Raiss $\cdot$ M. Scheibel}

\section{Langzeitergebnisse in der inversen Schulterendoprothetik}

\section{Zusammenfassung}

Hintergrund. Die inverse Endoprothetik gewinnt einen immer höheren Stellenwert in der Versorgung von degenerativen Erkrankungen, in der Frakturversorgung, bei posttraumatischen Folgezuständen und in der Revisions- und Tumorchirurgie des Schultergelenks.

Fragestellung. Fokus dieser Übersichtsarbeit ist die Evaluation von klinischen und radiologischen Langzeitergebnissen von inversen Schulterendoprothesen (iSTEP; Grammont-Typ, $155^{\circ}$ ). Dazu werden die funktionellen Scores, Bewegungsumfänge, Komplikationen und Standzeiten im Hinblick auf die Indikation verglichen.

Material und Methode. Klinische Studien, Diskussionen von neuesten Entwicklungen und Expertenempfehlungen werden ausgewertet.
Ergebnisse. Die iSTEP ist eine hervorragende Therapieoption für irreparable Rotatorenmanschetten-Massenrupturen (RM$M R)$, fortgeschrittene Defektarthropathien (CTA) und primäre Omarthrosen (OA) in Bezug auf Schmerzreduktion, Funktionsund Bewegungsverbesserung. Auch in der primären Frakturversorgung, der Revisionsund Tumorchirurgie profitieren Patienten von der Therapie mittels iSTEP. Trotz einer sehr hohen Rate an skapulärem Notching (SN) von bis zu $95 \%$ in Langzeitergebnissen erreichen die Patienten meist zufriedenstellende funktionelle Ergebnisse mit einem Constant Score von ca. 60 Punkten (Maximalwert $=100$ Punkte) im Durchschnitt. Obgleich ein Viertel der Patienten im Langzeitverlauf ein SN Grad 3-4 aufweisen, wird bei einer Revisionsrate von ca. $10 \%$ eine Standzeit von $90 \%$ nach 10 Jahren beschrieben. Auch wenn Bewegungsumfänge und Kraft nach ca. 8 Jahren nachlassen, bleibt die Patientenzufriedenheit unverändert. Die besten funktionellen Ergebnisse erreichen Patienten nach RM-MR, CTA und OA, während Patienten nach Revisionen und Frakturfolgezuständen schlechtere Resultate aufweisen. Schlussfolgerung. Die iSTEP liefert gute und zuverlässige klinische Ergebnisse in der Primärversorgung, als auch bei Revisionen. Trotz hoher Raten an SN profitieren die meisten Patienten auch im Langzeitverlauf (>10 Jahre) von der Operation.

\section{Schlüsselwörter}

Defektarthropathie - Omarthrose · Proximale Humerusfrakturen · Tumor · Revisionen

\section{Long-term results of reverse shoulder arthroplasty}

\section{Abstract}

Background. Reverse shoulder arthroplasty (RSA) is steadily gaining influence in the treatment of degenerative pathologies, fracture treatment posttraumatic situations, revision and tumor surgery of the shoulder. Objectives. Determination of subjective results and clinical function such as range of motion and complication of RSA (Grammont type, $155^{\circ}$ ) in long-term results in relation to indication.

Materials and methods. Evaluation of clinical studies with regard to long-term results, discussion of latest developments and expert opinions.

Results. RSA provides very good results for patients with irreparable massive rotator cuff tears, cuff-tear arthropathy and glenohumeral osteoarthritis with regard to pain relief and improvement in range of motion and function. Even patients suffering from proximal humerus fractures, fracture sequelae, tumors or in revision situations benefit from treatment with RSA. Although there is a high rate of scapular notching reported in the literature of up to $95 \%$ in long-term results, patients reach satisfying functional results with a mean Constant score of about 60 points (maximum $=100$ points). Although more than one quarter of patients display notching of grade 3 to 4 at long-term follow-up, RSA demonstrates a 10 -year survival of $90 \%$ with a revision rate of $10 \%$. While range of motion and strength seem to decline around 8 years after implantation, patients' satisfaction remains unaffected. Patients with massive rotator cuff tears, cuff-tear arthropathy and glenohumeral osteoarthritis achieve the best results, whereas patients after revision surgery and fracture sequelae perform worse. Conclusion. RSA provides reliable and good clinical results in primary surgery as well as in revisions. While scapular notching is frequently observed, patients still profit from this treatment in the long run.

\section{Keywords}

Cuff-tear arthropathy · Glenohumeral osteoarthritis - Proximal humerus fractures . Tumor $\cdot$ Revisions ein leichter Abfall sowohl hinsichtlich der Kraft als auch im CS erkennen [10]. Erstmals wurde dieser Trend in der Multicenterstudie von Favard et al. [11] beschrieben, wobei 8 Jahre nach der Implantation ein relativer Einbruch $\mathrm{zu}$ erkennen ist. Radiologische Auffälligkeiten, wie SN und „radiolucency lines" werden vermehrt nach 5 Jahren beobachtet. Alle 297 CTA-Patienten aus diesem Patientenkollektiv wiesen, beschrieben von Brunet et al. [12], nach über 8 Jahren (NU 60-239 Monate) eine signifikante Verbesserung im CS von $29( \pm 10)$ auf $63( \pm 4)$ Punkte, sowie im Bewegungsumfang (Flexion: $79^{\circ} \pm 34$ auf $138^{\circ} \pm 28$; Außenrotation: $8^{\circ} \pm 20$ auf $13^{\circ} \pm 21$ ) auf. Die Ergebnisse waren unabhängig vom SN, welches zu $65 \%$ (Grad 1-4; 26\% Grad 3-4) insgesamt, jedoch seltener nach „bony-increased offset reverse shoulder arthroplasty" (BIO-RSA; 15 vs. $37 \%$ ) auftrat.
Aufgrund der breiten Verteilung der finalen NU, haben Bassen et al. [10] ihre Gesamtkohorte (78\% CTA) in Subgruppen unterteilt. Die Patienten mit NU von 8-9 Jahren $(n=28), 9-10$ Jahren $(n=24)$ und über 10 Jahren $(n=22)$ wurden untereinander und mit den frühen NU verglichen. Bei den NU von 9-10 Jahren und jenen über 10 Jahren wurde verglichen mit den 5-Jahres-Ergebnissen eine signifikante Verschlechterung im CS ermittelt. 
Eine Erklärung für die Abnahmen des CS ist v.a. auf eine Krafteinschränkung durch die altersbedingte Ermüdung des M. deltoideus zurückführen, die auf unphysiologischen, biomechanischen Voraussetzungen des M. deltoideus basiert [8]. Die Muskelkontraktionen des M. deltoideus entsprechen aufgrund des adaptierten, medialisierten und distalisierten Rotationszentrums repetitiven und nicht-physiologischen Kontraktions-Dehnungs-Zyklen, welche die motorische Leistung verringern [13-15]. Bassens et al. [8] konnten daraufhin jedoch feststellen, dass Patienten eine unverminderte Zufriedenheit aufweisen, selbst wenn die Kraft und der Bewegungsumfang nachgelassen hatten.

\section{Omarthrose}

\section{Primäre Omarthrose}

Die genaue Pathogenese der primären degenerativen Schultergelenkerkrankung ist noch unvollständig eruiert. Ätiologisch wird eine multifaktorielle Genese mit Einfluss von Alter, genetischen Faktoren, Bindegewebseigenschaften und der mechanischen Belastung im Beruf sowie im Freizeitsport angenommen [16]. Die Gelenkpfanne kann anfangs konzentrisch degenerieren (Walch A1 und A2), weist jedoch meist einen frühen posterioren Substanzverlust (je nach Schweregrad des posterioren Substanzverlusts eingeteilt nach Walch B1, B2, B3) auf, der bis zur Subluxationsstellung führen kann [17, 18]. Klinisch stehen zunehmende Bewegungseinschränkungen mit Krepitationen bei Schmerzprogredienz im Vordergrund, die in einer funktionellen Schultersteife und sekundären RM-Insuffizienz resultieren können.

Während die Versorgung mit einer anatomischen Totalprothese anfangs einen höheren Stellenwert innehatte, gewinnt auch hier die iSTEP zunehmend an Einfluss. Moderne anatomische Implantate versprechen zukünftig Vorteile insbesondere bei jungen Patienten, doch sind sie nur bei leichten Glenoiddefekten Erfolg versprechend und begegnen bei schweren glenoidalen Substanzverlusten weiterhin großen Herausforderungen.
Erstmalig beschäftigten sich Mizuno et al. [19] mit der Versorgung von primären Omarthrosen (OA) bei intakter RM. Sie konnten zeigen, dass iSTEP bei posteriorer Subluxation (Walch B2) mittelfristig sehr gute klinische Ergebnisse ( $n=27$; CS: 76 Punkte; SN: $37 \%$ ) aufweisen und nahmen an, dass die Fixation mittels zentraler Schraube Vorteile hinsichtlich Stabilität und Langlebigkeit im Vergleich zur anatomischen Schultertotalendoprothesen (TEP) mit zementierter Glenoidkomponente aufzeigen könnte.

Die französische Multicenterstudie von Collin et al. [20] beschreibt erstmalig Langzeitergebnisse einer Patientenkohorte mit OA (Walch B1, B2, B3, C) nach durchschnittlich 7,7 (5-16) Jahren: 45 Patienten mit 49 Schultern. Der CS verbesserte sich präoperativ von 30 $( \pm 12)$ auf $68( \pm 13)$ Punkte, der aCS von $43( \pm 19)$ auf $105( \pm 19)$. Es wurde eine Flexion von $145^{\circ}( \pm 26)$, Außenrotation von $18^{\circ}( \pm 14)$ und durchschnittliche Innenrotation höher LWK3 erreicht. Patienten, deren posteriorer Substanzverlust mithilfe eines Knochenspans aufgefüllt worden war (33\%), zeigten kein signifikant besseren CS auf. Innerhalb der verschiedenen Glenoidmorpholgien gab es keine signifikanten Unterschiede auch wenn Patienten mit einem Walch-B2Glenoid die besten Ergebnisse erreichten. Trotz einer moderat hohen Rate an SN (43\%), erreicht diese Patientenkohorte mit OA die besten klinischen Ergebnisse im Vergleich zu allen anderen Indikationen (• Tab. 1).

Bacle et al. [8] konnten in ihrer heterogenen Kohorte von 87 nachuntersuchten Patienten 9 OA-Patienten verzeichnen. Hier verbesserte sich der CS von präoperativ $26( \pm 11)$ auf $70( \pm 6)$ in mittelfristigen Ergebnissen und $62( \pm 8)$ Punkte bei einer Flexion von $140^{\circ}( \pm 27)$ nach über 10 Jahren.

\section{Rheumatoide Arthritis}

Anders als bei der Omarthrose stehen bei der rheumatoiden Arthritis (RA), neben der durch jahrelange Kortikosteroideinnahme herabgesetzten Knochenqualität, v. a. die horizontale und kraniale Erosion der Schulterpfanne im Vordergrund.
Aufgrund der starken Glenoiderosion wird die ohnehin bei Rheumatikern schlechte Sehnenqualität der RM weiter belastet und führt zur sekundären RMInsuffizienz. Infolge dessen können RMMR entstehen, die zu Erosionen am korakoakromialen Bogen führen. Sie ähneln einer Humeruskopfdezentrierung nach kranial wie bei CTA. Patienten, die eine sehr fortgeschrittene Progredienz ihrer rheumatoiden destruktiven Gelenkerkrankung aufweisen, zeigen RM-Rupturen sowie sehr geringe Knochenmasse auf und wurden erstmals von Neer als „End-stage-Patienten“ beschrieben und stellen eine besondere Herausforderung für Chirurgen dar [21].

Woodruff et al. [22] untersuchten in ihrer retrospektiven Studie 12 Frauen (13 Schultern; durchschnittliches Alter: 64 [43-72] Jahre) nach durchschnittlich 87 (60-110) Monaten. Ausgeschlossen wurden $12 \%$ der Patienten aufgrund eines Schraubenbruchs vor der letzten NU, $18 \%$ zeigten geringe Lockerungszeichen und weitere $18 \%$ zeigten bei letzten Röntgenaufnahmen eine komplette Glenoidlockerung auf. Der durchschnittliche CS lag bei 69,0 (95\%-Konfidenzintervall [-KI] 50,7-64,3) Punkten, $77 \%$ (10/13) waren zum Zeitpunkt der letzten NU schmerzfrei. Die Autoren resümierten, dass die iSTEP gute Schmerzbefreiung der Patienten bei sehr variablen funktionellen Ergebnissen liefert.

Lévigne [23] konnte in seiner Kohorte bei 34 Patienten und 39 iSTEP nach 92 (60-147) Monaten zufriedenstellende und reproduzierbare Ergebnisse (CS: 63 Punkte; Flexion $140^{\circ}$; Außenrotation $13^{\circ}$; Innenrotation LWK3; SSV: $76 \%)$ verzeichnen. Davon waren 6 sog. End-stage-Patienten, welche mit einer BIO-RSA komplikationslos versorgt wurden und eine überdurchschnittlich große Verbesserung zu präoperativen Ergebnissen aufweisen konnten (CS: 72 Punkte; Flexion $138^{\circ}$; Außenrotation $22^{\circ}$; Innenrotation LWK3; SSV: $90 \%$ ). 8 Patienten erreichten eine NU von über 10 Jahren (120-147 Monate) und wiesen vergleichbare Ergebnisse auf (CS: 57 Punkte; Flexion 129\%; Außenrotation $1^{\circ}$; Innenrotation LWK3; SSV: $68 \%$ ). Insgesamt wurden abgesehen von den zwei Revisionen (aufgrund eines Infekts) 


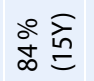

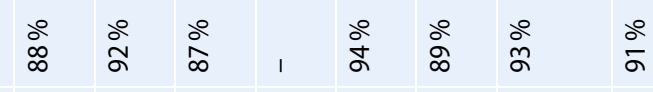

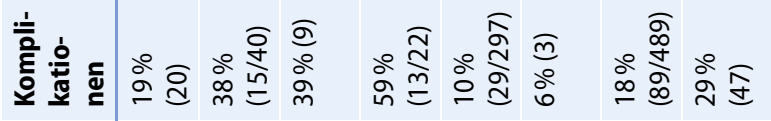

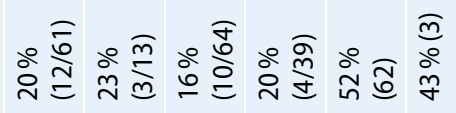

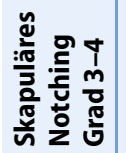

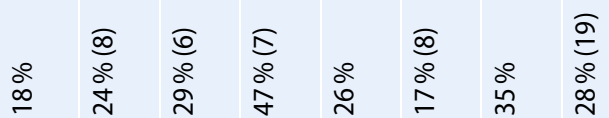

ஃ̊

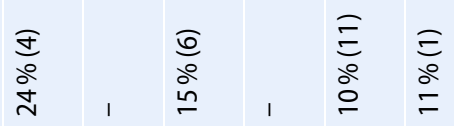

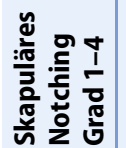

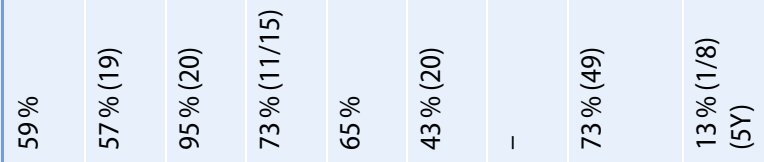

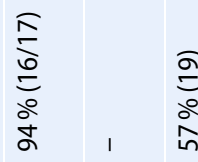

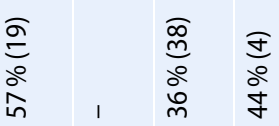

খั๊

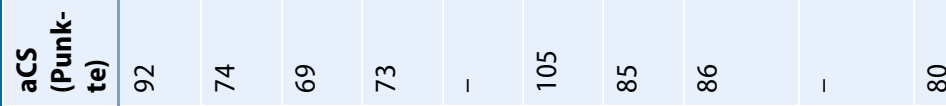

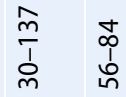

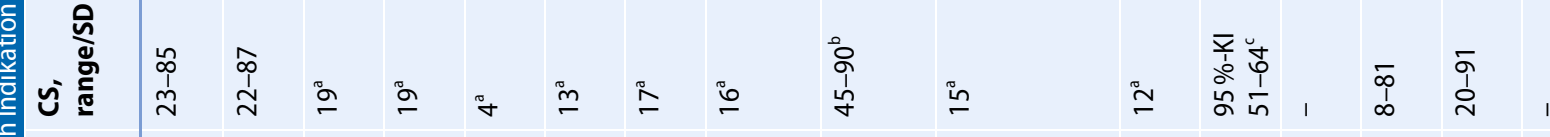

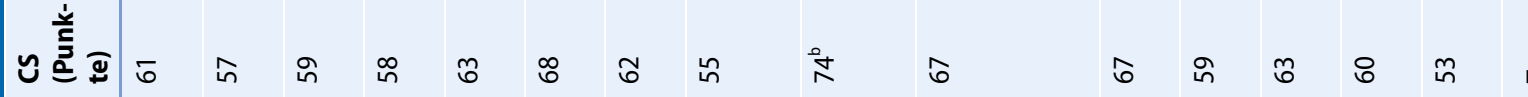

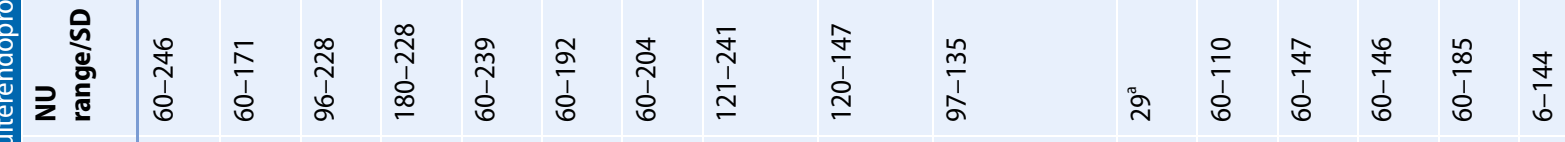

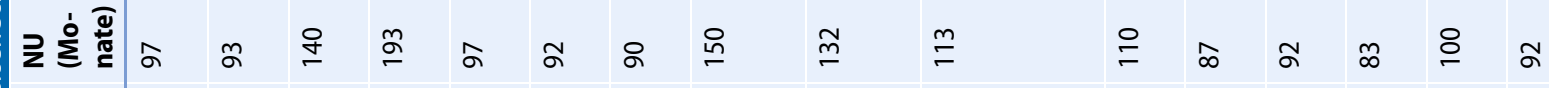

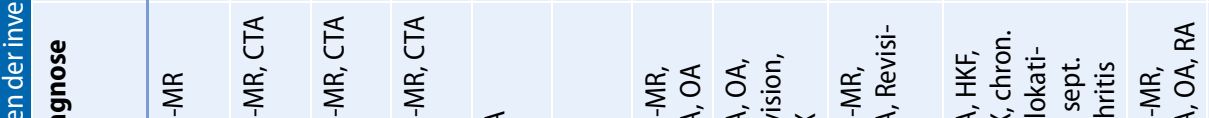

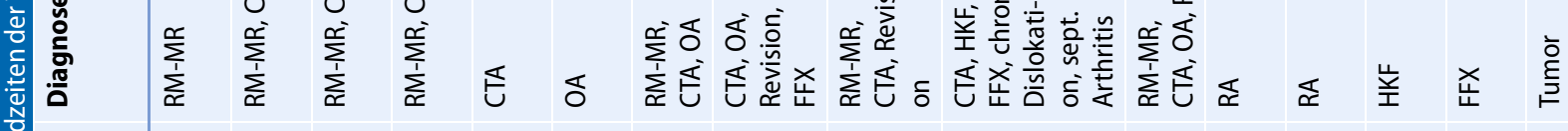

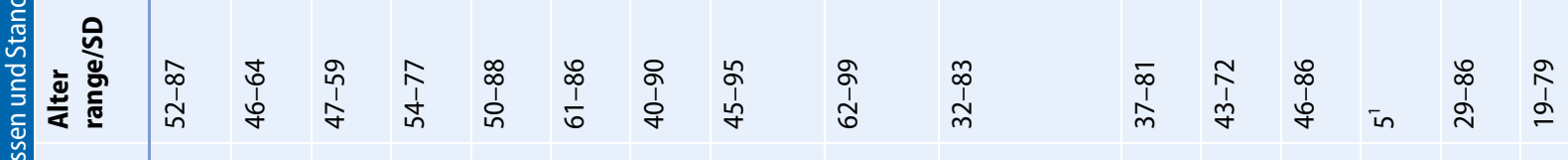
产要焉

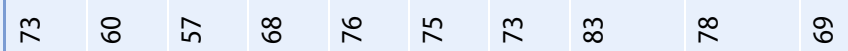

m

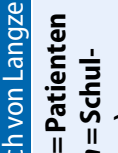

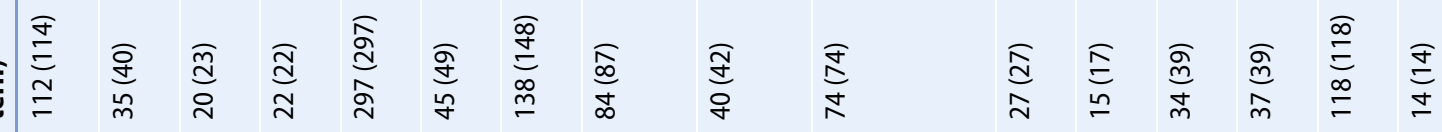

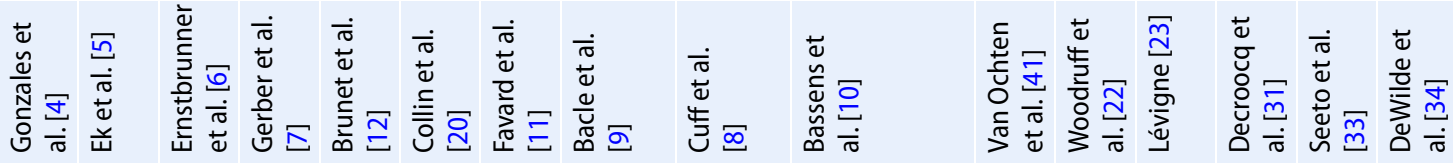




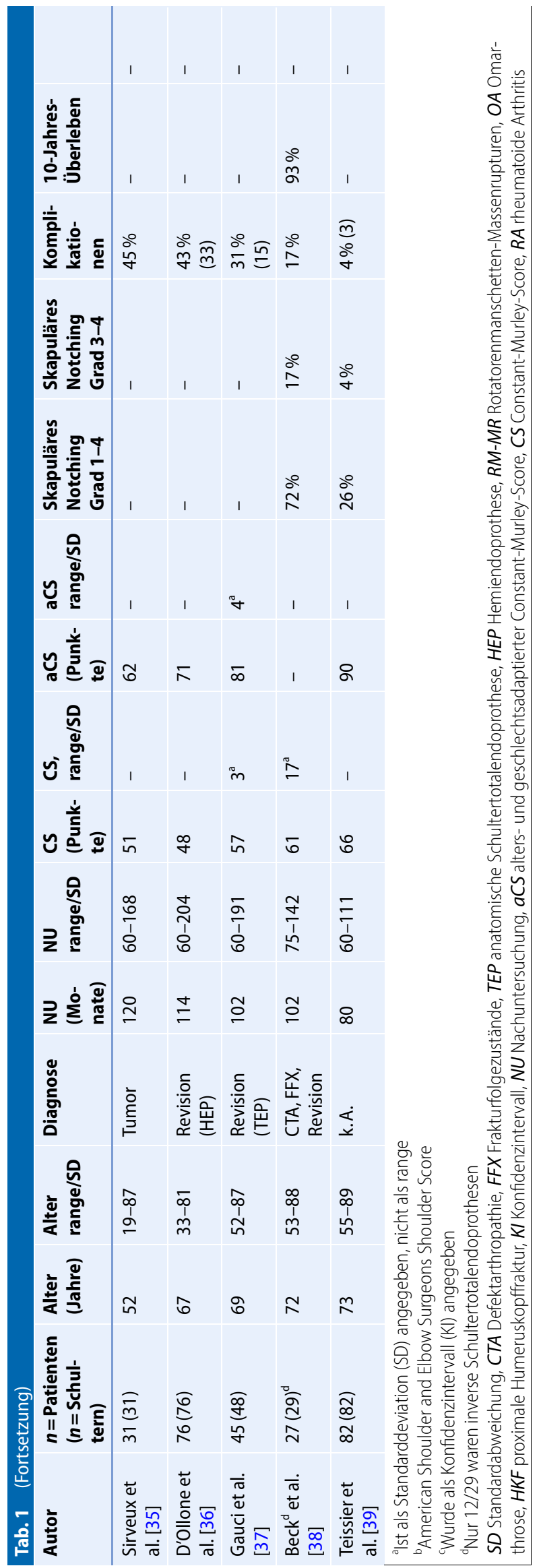

keine Lockerungszeichen beobachtet; SN wurde zu $57 \%$ beschrieben. Diese Studie bestätigt die guten Langzeiterbnisse nach RA und beschreibt keine signifikanten Unterschieden im Verlauf zu den kurzfristigen Ergebnissen dieser Kohorte nach 2 Jahren.

\section{Proximale Humerus- kopffrakturen}

\section{Akute Frakturen}

Akute proximale Humeruskopffrakturen (HKF) sind neben Frakturen des Oberschenkelhalses und des distalen Radius, die dritthäufigste Frakturentität bei älteren Menschen [24]. Eine chirurgische Intervention ist insbesondere bei Dislokation der Knochenfragmente indiziert (ca. $20 \%$; [25]). Obwohl die anatomische Rekonstruktion mittels Plattenosteosynthese (ORIF) oder anatomischer HEP lange Goldstandard war, überzeugte die iSTEP auch hier aufgrund zuverlässigerer und besserer klinischer Ergebnisse mit geringeren Revisionsraten bei grob dislozierten 3- und 4-Part-Frakturen [26-28]. Bei der sog. Head-split-Fraktur, einer seltenen Entität (ca. $1 \%$ aller Frakturen) der HKF, führt alleine die iSTEP zu zufriedenstellenden Ergebnissen im Vergleich zu HEP und ORIF [29].

In der retrospektiven Studie von Cazaneuve et al. [30] wurden $35 \mathrm{~Pa}$ tienten (33 Frauen, durchschnittliches Alter: 75 [58-92] Jahre) eingeschlossen. Die durchschnittliche NU nach 86 (12-204) Monaten erreichte einen CS von 53 Punkten, der damit 2 Punkte unter dem nach 12 Monaten erhobenen CS von 55 (20-84) Punkten lag. Im Vergleich zu der 1-Jahres-NU verloren die Patienten im Durchschnitt einen Punkt bei der Kraft und einen auf der Schmerzskala; der Bewegungs- und der Rotationsumfang blieben unverändert. Damit lag der CS im Vergleich zur gesunden, kontralateralen Seite bei $69 \%$. Bei $23 \%$ traten Komplikationen auf, $17 \%$ mussten daraufhin revidiert werden. Trotz langer chirurgischer Erfahrung beschreiben die Autoren 50\% SN bei der Versorgung mit Delta IIIProthesen (DePuy Synthes Inc., Warsaw, Indiana, USA). Sie empfehlen eine 
Versorgung dieser aufgrund der hohen Komplikationsrate daher nur bei osteoporotischen Patienten über 70 Jahren auf der nicht-dominaten Seite. Vermutlich beeinflussen auch Frakturmorphologie und Versorgungszeitpunkt nach Trauma das endgültige funktionelle Ergebnis.

Decroocq et al. [31] beschreiben zufriedenstellende Ergebnisse (CS: 60 Punkte; Flexion: $128^{\circ}$; Außenrotation: $12^{\circ}$ ) mit einem frakturspezifischem Schaft bei 39 Patienten, trotz des hohen Alters (77 \pm 5 Jahre) und Osteoporose (95\% Frauen), sowie Non-union des Tuberculum majus in $23 \%$ der Fälle. Eine erfolgreiche Konsolidation des Tuberculum majus korreliert statistisch signifikant mit einer verbesserten Außenrotation.

\section{Frakturfolgezustände}

Auch Patienten mit initial konservativ oder osteosynthetisch versorgten HKF können im Verlauf - trotz adäquater Erstversorgung - infolge von Infektionen, Humeruskopfnekrosen, sekundären RM-Insuffizienzen oder Gelenkdestruktionen einer Versorgung mittels iSTEP bedürfen.

Bacle et al. [9] haben 10 Patienten (11\%) mit Frakturfolgezuständen (FFX) nachuntersucht. Bei einer durchschnittlichen Flexion von $119^{\circ}( \pm 31)$ stieg der CS von präoperativ $27( \pm 8)$ auf 55 $( \pm 20)$ in den mittelfristigen Ergebnissen und letztlich auf $45( \pm 22)$ nach über 10 Jahren. Die Kraft lag bei der finalen NU bei knapp unter $2 \mathrm{~kg}$, und die Patienten erreichten nur $20( \pm 11)$ von maximal 40 Punkten im Bewegungsumfang. Nach Revisionen erreichen Patienten mit FFX hier die schlechtesten klinischen Ergebnisse. Bassens et al. [10] konnten 5 Patienten (7\%) mit Malunion nach HKF und 3 (4\%) mit avaskulärer Nekrose einschließen. Hier lag der aCS nach mindestens 8 Jahren bei 74,5 ( \pm 21$)$ Punkten. Wegen der sehr überschaubaren Patientenzahlen und des heterogenen FFX-Patientenkollektivs können Schlussfolgerungen für die Versorgung mittels iSTEP nur bedingt gezogen werden. Trotzdem erzielen iSTEP bei FFX keine zuverlässigen klinischen Ergebnisse mit einer weiten Streuung und erreichen im Vergleich $\mathrm{zu}$ allen anderen Indikationen in dieser Studie, sowohl im Vergleich zu akuten Frakturen ( $n=3$; aCS: $79 \pm 11$ Punkte) als auch bei chronischen Dislokationen $(n=3$; aCS: $75 \pm 23$ Punkte) und septischen Arthritiden ( $n=2$; aCS: $77 \pm 23$ Punkte), die schlechtesten Ergebnisse.

Seeto et al. [32] beschreiben eine Komplikationsrate von $73 \%$ bei Typ3- und sogar zu $94 \%$ bei Typ-4-FFX nach der Boileau-Klassifikation mit einer Revisionsrate von $33 \%$ (Typ 3) und $24 \%$ (Typ 4), wovon bei 2 Patienten die iSTEP explantiert werden musste. Die Komplikationsrate nach Typ-1- und Typ-2-FFX lag bei einem Viertel und die Notwendigkeit zur Reoperation bei $0 \%$ (Typ 1) bzw. 1 von 7 Patienten (Typ 2). Nichtsdestotrotz wurden von insgesamt 118 Patienten nach gut 8 Jahren relativ ähnliche durchschnittliche, funktionelle Ergebnisse (CS: Typ 1: 56 Punkte, Typ 2: 41 Punkte, Typ 3: 53 Punkte, Typ 4: 51 Punkte; SSV: Typ 1: $68 \%$, Typ 2: $42 \%$, Typ 3: $57 \%$, Typ 4: $62 \%$ ) bei einer sehr großen Streuweite (CS: 20-91 Punkte; SSV: 0-100\%) erreicht. Während die präoperative Steife und Beweglichkeit keinen Einfluss auf das finale Ergebnis hatte, so weisen Patienten mit zuvor gescheiterten Fixationsversuchen eine erhöhte Rate an Komplikationen ( $37 \%$ vs. $27 \%$ ), Infektionen ( $2 \%$ vs. $0 \%$ ) und Reoperationen (17\% vs. $8 \%$ ) auf. Das Fehlen des Tuberculum majus ist ein prognostisch prädiktiver Faktor für einen signifikant schlechteren CS (50 vs. 56 Punkte), schlechtere Außenrotation $\left(-4^{\circ}\right.$ vs. $\left.12^{\circ}\right)$, ein Außenrotations-LagZeichen (67\% vs. $12 \%$ ) sowie humerale Lockerung (56\% vs. $11 \%$ ). Auch wenn Patienten mit FFX von iSTEP profitieren können, weisen diese mit Voroperatioenen und fehlendem Tuberculum majus ein erhöhtes, nicht insignifikantes Risiko für Komplikationen und Lockerungen auf [33].

\section{Tumor}

Der proximale Humerus ist die dritthäufigste Lokalisation von primären Knochen- und Weichteiltumoren. Die Rekonstruktion und der Funktionserhalt der oberen Extremität gestaltet sich nach der Humerusresektion als schwierig, sobald die RM vom Tumor infiltriert wird. Diese RM-Resektion hat, ähnlich wie bei der CTA, einen pseudoparalytischen Arm zu Folge und kann mittels iSTEP, gegebenenfalls kombiniert mit einem Sehnentransfer, eine funktionelle Verbesserung erzielen.

DeWilde et al. [34] haben insgesamt 14 Patienten mit einem Tumor des proximalen Humerus mittels iSTEP versorgt. Zwei der Patienten wurden an einem anderen Krankhaus von einem anderen Operateur und mit einem kombinierten Latissimus-dorsi- und Teres-major-Muskeltransfer versorgt. Insgesamt konnten 7 der 12 Patienten mit konventioneller iSTEP nach durchschnittlich über 9 Jahren nachuntersucht werden; die beiden Patienten mit kombiniertem Muskeltransfer wurden nur ein Jahr nach ihrer Operation nachuntersucht. Bei einer Revisionsrate von $16 \%$ lag der durchschnittliche CS bei 78 Punkten. Die aktive Abduktion lag bei $163^{\circ}\left(90-180^{\circ}\right)$, die beiden Patienten mit Muskeltransfer erreichten dabei nur $135^{\circ}\left(120-150^{\circ}\right)$; die aktive Außenrotation lag bei beiden Patienten mit Muskeltransfer bei $60^{\circ}$ in Abduktion, während die $7 \mathrm{mit}$ konventionellen iSTEP versorgten Patienten nur $17^{\circ}$ erreichten. Der Score für allägliche Aktivitäten, die eine Außenrotation voraussetzen (ADLER-Score), wurde erhoben. Er lag bei 27,5 (26-26) vs. 23,6 (14-28) Punkten zugunsten der beiden Patienten mit Muskeltransfer.

Auch wenn diese Studie aufgrund ihrer Limitationen (geringe Fallzahl, verschiedene Operationstechniken, verschiedene NU-Zeiträume) keine statistische Analyse erlaubt, beschreibt sie gute funktionelle Ergebnisse. Die Autoren diskutieren, dass es alleine mithilfe des M. deltoideus nicht möglich ist, Aktivitäten des alltäglichen Lebens, die eine (Außen-)Rotationsbewegung voraussetzen, suffizient auszuführen. Somit kann die Kombination mit einem Muskeltransfer ein möglicher Ansatz sein, die insuffizienten Außenrotatoren $\mathrm{zu}$ stärken.

Sirveux et al. [35] konnten $13 \mathrm{~Pa}$ tienten nachuntersuchen, bei denen aufgrund eines primären Tumors oder einer Metastase eine Humerusresektion durchgeführt wurde und zusätzlich 
Hier steht eine Anzeige.

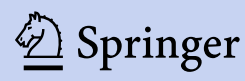


mit einer iSTEP versorgt wurden. Nach durchschnittlich 10 Jahren lag der CS bei 51 Punkten und es wurde eine Flexion von $113^{\circ}$ erreicht. In allen Fällen wurde der Humeruskopf mitsamt tendinöser Ansätze mit einer durchschnittlichen Länge von $11(3-20) \mathrm{cm}$ reseziert. In Folge dessen wurden alle Patienten mit einem Langschaft versorgt und erhielten teilweise zusätzlich einen Zementspacer, eine individuelle metaphysäre Prothesenanfertigung oder einen gefrorenen Allograft zur Stabilisierung.

Isolierte Schäfte können nur bei kleinen humeralen Defekten zum Einsatz kommen. Die einzige Patientin, die mit kurativem Therapieansatz mit iSTEP versorgt worden war, zeigte nach 14 Jahren Schmerzen und ROM-Verlust nach humeraler Lockerung und ein Notching Grad 4 (CS: 15 Punkte). Die zusätzliche Stabilisierung mithilfe des Zementspacers zeigte auch nach 10 Jahren keine Lockerung auf und ließ sich mit einem Latissimus-dorsi-Transfer kombinieren (CS: 62 Punkte; Flexion: 113º).

Bei der Versorgung mit Allografts sollte auf Zement in der Knochen-AllograftSchnittstelle verzichtet werden und zur Kompression gegebenenfalls eine laterale Platte angebracht werden, um das Risiko einer Non-union und möglich folgender Dislokation zu minimieren. Eine erhöhte Infektionsgefahr wurde nicht dokumentiert, wenn auch die Resorption des Allografts beobachtet werden konnte (CS: 52 Punkte; Flexion: $108^{\circ}$ ). Patienten mit einer individuell angefertigten Endoprothese waren mit durchschnittlich 38 Jahren deutlich jünger, zeigten jedoch initial eine hohe Rate an humeralen Lockerungen $(30 \%)$ auf, die nach Versorgung mithilfe eines Allografts erfolgreich stabilisiert werden konnten und letztendlich gute Funktion sowie nach Latissimusdorsi-Transfer gute Außenrotation aufweisen konnten (CS: 68 Punkte; Flexion: $\left.150^{\circ}\right)$.

\section{Revisionen}

Häufige Anwendung findet die iSTEP in der Revision von aseptischen oder infektassoziierten Lockerungen von anatomischen HEP und TEP. Des Weiteren führt eine sekundäre RM-Insuffizienz nach
HEP oder TEP zu Bewegungseinschränkungen bis hin zu pseudoparalytischen Ausfällen, die ebenfalls von einem Wechsel auf iSTEP profitieren. Bei Infektionen müssen alle körperfremden Elemente explantiert und das Gelenk saniert und antibiotisch versorgt werden. Ein einzeitiger vs. zweizeitiger Wechsel muss je nach Weichteilsituation und Keimspektrum, sowie vorhandener biofilmaktiver antibiotischer Therapie abgewogen werden. Hierbei ist die Überbrückung mit einem antibiotikabeladenen Spacer die sicherere Wahl zur Keimeliminierung.

D’Ollonne et al. [36] konnten $76 \mathrm{~Pa}$ tienten mit revidierter HEP nach fast 10 (5-17) Jahren nachuntersuchen, welche initial aufgrund einer HKF (66\%), OA (17\%), FFX (12\%) und CTA (5\%) versorgt worden waren. Durchschnittlich folgte die iSTEP 48 (35-201) Monate nach primärer Intervention und verbesserte den CS von 32 auf 48 Punkte, den SSV von $10 \%$ auf $60 \%$. und die Flexion von $57^{\circ}$ auf $107^{\circ}$. Die Migration, Lyse oder Malunion des Tuberculum majus stellt sich auch hier als prädiktiver negativer Faktor heraus und führt zu schlechteren ROM und Funktion (aCS: $66 \%$ vs. $82 \%$; Flexion: $100^{\circ}$ vs. $123^{\circ}$; Außenrotation: $1^{\circ}$ vs. $\left.10^{\circ}\right)$.

Gauci et al. [37] haben 48 Patienten mit versagter TEP nach fast 9 (5-16) Jahren nachuntersucht. Der CS verbesserte sich von $31( \pm 3)$ auf $57( \pm 3)$ Punkte, der SSV von $22( \pm 5) \%$ auf $65( \pm 4) \%$, bei einer Flexion von $124^{\circ}( \pm 5$; präoperativ: $80^{\circ} \pm 10$ ), jedoch einer Verschlechterung der Außenrotation und Stagnation der Innenrotation.

Die Ergebnisse nach Revisionseingriffen wurden auch von Bacle et al. ([9]; $n=21$ ) und Cuffet al. ([8]; $n=10)$ anhand vereinzelter Patienten nach gescheiterter TEP analysiert.

Bei Cuff et al. [8] verbesserte sich der ASES von 27 (0-63) auf 72 (37-97) und bis hin zu 68 (48-83) Punkten. Bei einer Abduktion von $89^{\circ}(0-150)$ und einer aktiven Außenrotation von $14^{\circ}$ (-30-90) lag die durchschnittliche Flexion bei $98^{\circ}$ (0-180).

Bei Bacle et al. [9] verbesserte sich der CS von präoperativ $21( \pm 13)$ auf mittelfristig $55( \pm 16)$ bis hin $\mathrm{zu} 45( \pm 17)$ Punkten nach über 10 Jahren. Es wurde im Vergleich zu den mittelfristigen Ergebnissen eine signifikante Verschlechterung in Flexion $\left(128^{\circ}\right.$ auf $\left.116^{\circ}\right)$ sowie in der CS-Subkategorie Mobilität (22 auf 18) und eine Halbierung der Kraft (2,7 auf $1,35 \mathrm{~kg}$ ) festgestellt. Die Kohorte der Revisionen in dieser Studie war die Einzige, bei der eine signifikante Abnahme in den CS-Subkategorien Schmerz und Aktivitäten zu verzeichnen war. In dieser Studie erreichen Patienten nach Revisionen nach über 10 Jahren die schlechtesten funktionellen Ergebnisse im Vergleich zu CTA, OA, FFX und RM-MR und wiesen nach 10 Jahren die geringste Abduktionskraft auf.

\section{Schaftlose Endoprothesen}

Während in der gesamten Orthopädie ein Trend zu knochensparenden Implantaten besteht und sich erste Modelle in anatomischer Schultertotalendoprothetik bewährt haben, sind im letzten Jahrzehnt immer mehr schaftlose iSTEP zu finden. Schaftlose Komponenten haben einen großen Vorteil bei humeralen Achsendeviationen und können daher in posttraumatischen Situationen und bei metaphysären Deformitäten eingesetzt werden.

In der Studie von Beck et al. [38] wurden 49 Schultern (bei 47 Patienten) operiert. Nach 102 (75-142) Monaten konnten 29 Prothesen (59\%) eingeschlossen werden, davon erhielten jedoch nur 12 ein schaftloses Implantat; in 17 Fällen war ein zusätzlicher Kurzschaft nötig, um primäre Stabilität bei schlechter Knochenqualität oder metaphysären Knochendefekten zu garantieren (7 CTA, 7 FFX, 3 Revisionen). Die schaftlose, humerale Komponente konnte trotz ihrer theoretischen Vorteile bei Achsendeviationen in posttraumatischen Situationen nur bei CTA-Patienten Verwendung finden. Nichtsdestotrotz werden gute klinische Langzeitergebnisse mit einem CS von $60,5( \pm 16,8)$ Punkten, einer Flexion $135,5^{\circ}\left( \pm 26,6^{\circ}\right)$ und einer Schmerzreduktion von $7,5( \pm 1,2)$ auf 1,4 $( \pm 1,5)$ Punkten in der visuellen analogen Schmerzskala erzielt. Nach 8,4 Jahren erreichte die TESS trotz einer Komplikationsrate von $17 \%$ und einer Rate an SN von $72 \%$ (17\% Grad 3) eine Stand- 
zeit von $93 \%$. Schaftlose Komponenten erreichen ähnlich gute Ergebnisse wie Kurzschäfte.

Teissier et al. [39] konnten 82 schaftlose Implantate mit einer NU von über 6 (5-10) Jahren einschließen. Die finale Flexion verbesserte sich von $96^{\circ}\left(0-160^{\circ}\right)$ auf $143^{\circ}\left(90-170^{\circ}\right)$, Abduktion von $89^{\circ}$ $\left(0-160^{\circ}\right)$ auf $139^{\circ}\left(80-160^{\circ}\right)$, Außenrotation von $26^{\circ}\left(-60-70^{\circ}\right)$ auf $39^{\circ}\left(20-70^{\circ}\right)$, CS von 40 (12-76) auf 68 (37-100) Punkte bei einem Kraftzuwachs von $2(0-7)$ auf $6(2-12) \mathrm{kg}$. Es wurden keine Lockerungszeichen und ein $\mathrm{SN}$ in $26 \%$ beobachtet. Die Autoren schlussfolgerten, dass schaftlose Implantate eine gute primäre Stabilität und Integration in den Knochen gewährleisten.

Auch wenn schaftlose Komponenten Revisionen in Zukunft erleichtern können, wird in bestimmten Fällen mit schlechter Knochenqualität eine Kurzschaftalternative unentbehrlich bleiben.

\section{Diskussion}

Die iSTEP nach Grammont weist gute Langzeitergebnisse auf, auch wenn Patienten mit einer Defektarthropathie und einer Omarthrose eine deutlich bessere Funktion nach der Versorgung aufzeigen als solche mit einem Frakturfolgezustand oder nach einer Revision. In der Versorgung von akuten Frakturen sowie bei irreparablen RM-MR kommt die iSTEP auch regelmäßig bei jüngeren Patienten zum Einsatz und beweist ihre Funktionsfähigkeit. Selbst in der Tumorchirurgie kann die iSTEP Patienten nach radikalen RM-Resektionen zu Beweglichkeit und Kraft verhelfen.

Ascione et al. [40] beschrieben bei 1035 implantierten iSTEP nach mindestens 5 Jahren insgesamt eine Komplikationsrate von $18,7 \%$. Humerale Komplikationen treten $\mathrm{zu} 3,3 \%$ auf und haben einen negativen Effekt auf das funktionelle Endergebnis. Sie treten jedoch vermehrt bei Patienten mit Revisionen und Tumoren auf.

Cuff et al. [8] und Bacle et al. [9] konnten mittels Kaplan-Meier-Kurve nach 10 Jahren eine Standzeit von $>90 \%$ aufzeigen (91\% und $93 \%$ ) und verzeichnen dabei eine Revisionsrate von ca. einem Viertel. Ein großes Problem, das zu Komplikationen führen kann, ist das SN. Bei bis zu $95 \%$ wird SN in Langzeitergebnissen beschrieben. Davon schlägt durch mechanische Knochenerosion bei bis zu $47 \%$ die mediale Schaftkomponente bis zur ersten Schraube am Glenoid an (• Tab. 1).

Favard et al. [11] beschrieben eine Standzeit der iSTEP von $89 \%$ nach 10 Jahren. $72 \%$ der Patienten erreichten einen CS von $>30$ Punkten. Bassens et al. [10] zeigen nach 8 Jahren noch eine Standzeit von $97 \%$ mit einer Revisionsrate von $3 \%$ auf. Mehrere Studien beschreiben einen Funktions- und Kraftverlust nach ca. 8 Jahren [9-11]. Um die motorische Leistung des $\mathrm{M}$. deltoideus in dieser unphysiologischen, biomechanischen Situation beim alternden Patienten zu evaluieren, müssen weitere Studien durchgeführt werden.

Van Ochten et al. [41] konnten mit einer durchschnittlichen NU von 9,2 $( \pm 2,4)$ Jahren bei 27 primär implantierten iSTEP (48\% RM-MR, $11 \%$ CTA, $22 \%$ OA, 19\% RA) eine Standzeit von $82,4 \%$ insgesamt und 98,3\% für die unzementierte, humerale Komponente über 12,5 Jahre beschreiben. SN wurde in $94 \%$ beobachtet - davon 24\% Grad 3-4.

Patienten mit RM-MR, CTA und OA konnten die besten klinischen Ergebnisse erreichen, während Patienten, insbesondere nach akuten Frakturfolgezuständen und nach Revisionen aber auch im Fraktur-, Tumorsetting und bei entzündlichen Arthritiden im Vergleich deutlich schlechter abschneiden (• Tab. 1).

Gerber et al. [7] beschrieben in ihrer Kohorte von RM-MR eine Standzeit von $87 \%$ nach 10 und $84 \%$ nach 15 Jahren.

Neueste Entwicklungen ermöglichen neben knochensparenden, schaftlosen Komponenten v.a. eine Lateralisierung des Drehzentrums. Mit Hilfe eines knöchernen oder metallischen Aufbaus (glenoidal sowie humeral) mittels steilerem „neck-shaft angle“ (145 ${ }^{\circ}$ und $\left.135^{\circ}\right)$, gebogener Schäfte oder Onlay-Design soll SN verhindert werden. Die Lateralisierung des Drehzentrums führt auf Kosten eines verminderten Hebelarms $\mathrm{zu}$ einer erhöhten Rekrutierung von Muskelfasern und zu einer natürlicheren Schulterkontur. Diese Rekrutierung insbesondere von anterioren und poste- rioren Fasern des M. deltoideus soll die Innen- sowie Außenrotation verbessern und somit die verbesserte selbständige Ausführung von alltäglichen Bewegungen ermöglichen (Zähneputzen, Anund Auskleiden, Haare kämmen, Toilettenhygiene etc.). Allerdings erhöht die Lateralisierung des Drehzentrums auch die Scherkräfte, wodurch vermehrt Ermüdungsfrakturen der Spina scapulae zu beobachten sind. Große Glenosphären und inferiorer Tilt verringern ebenfalls den mechanischen Zusammenstoß zwischen medialer Schaftkomponente und inferioren Schrauben, und könnten demnach dazu beitragen die Lockerungsrate $\mathrm{zu}$ minimieren und Standzeiten zu verbessern.

Da sich alle hier aufgeführten Studien mit der iSTEP nach Grammont auseinandersetzen, wird es noch einige Jahre dauern, bis diese Neuerungen des Designs auch die ersten Langzeitergebnisse hervorbringen. Weiterhin gilt es $\mathrm{zu}$ evaluieren, ob diese Verbesserungen der Biomechanik in der Theorie auch zu einer verbesserten Rotationsbeweglichkeit und weniger SN sowie besseren Bedingungen für den M. deltoideus führen oder ob andere Komplikationen die Erfolgsaussichten schmälern.

\section{Fazit für die Praxis}

\footnotetext{
- Patienten nach Rotatorenmanschetten-Massenrupturen (RM-MR), Defektarthropathie (CTA) und Omarthrose (OA) scheinen die besten Indikationen mit sehr guten klinischen Ergebnissen darzustellen.

- Patienten mit Frakturfolgezuständen (FFX) erzielen schlechtere Ergebnisse in der sekundären Versorgung mittels iSTEP, ähnlich der Patienten nach Revisionseingriffen.

- Die Stagnation im Constant-Murley-Score (CS) ist bedingt durch die Abnahme der Kraft, die auf unphysiologischen, biomechanischen Voraussetzungen des M. deltoideus basiert.

- Es wird eine Standzeit von >90\% nach 10 Jahren aufgezeigt.
} 


\section{Korrespondenzadresse}

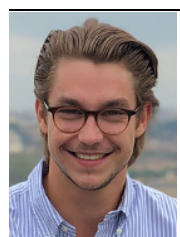

Jan-Philipp Imiolczyk

Centrum für Muskuloskeletale

Chirurgie (CMSC), Charité-

Universitätsmedizin Berlin

Augustenburger Platz 1,

13353 Berlin, Deutschland

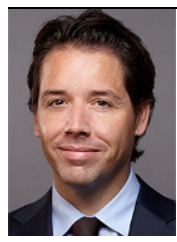

Prof. Dr. Markus Scheibel

Centrum für Muskuloskeletale Chirurgie (CMSC), CharitéUniversitätsmedizin Berlin Augustenburger Platz 1, 13353 Berlin, Deutschland markus.scheibel@charite.de

\section{Einhaltung ethischer Richtlinien}

Interessenkonflikt. J.-P. Imiolczyk und F. Freislederer geben an, dass kein Interessenkonflikt besteht. P. Raiss und M. Scheibel sind Berater für Wright Medical Inc.

Für diesen Beitrag wurden von den Autoren keine Studien an Menschen oder Tieren durchgeführt. Für die aufgeführten Studien gelten die jeweils dort angegebenen ethischen Richtlinien.

\section{Literatur}

1. Boileau P, Watkinson DJ, Hatzidakis AM, Balg F (2005) Grammont reverse prosthesis: design, rationale, and biomechanics. J Shoulder Elbow Surg 14(1):S147-S161

2. Kim SH, Wise BL, Zhang Y, Szabo RM (2011) Increasing incidence of shoulder arthroplasty in the United States. J Bone Joint Surg Am 93(24):2249-2254

3. Hamada K, Fukuda H, Mikasa M, KobayashiY (1990) Roentgenographic findings in massive rotator cuff tears. A long-term observation. Clin Orthop Relat Res 254:92-96

4. Gonzalez J-F, Fountzoulas K, Chelli M, Seeto B, Boileau P (2016) Reverse shoulder arthroplasty for non-operated, irreperable massive cuff tear (Hamada I-II-III) minimum 5-year follow up. In: Boileau P, Walch G, Molé D, Favard L, Lévigne C, Sirveaux $F$ et al (Hrsg) Shoulder concepts: reverse shoulder arthroplasty. Sauramps Medical, Montpellier, S71-80

5. Ek ET, Neukom L, Catanzaro S, Gerber C (2013) Reverse total shoulder arthroplasty for massive irreparable rotator cuff tears in patients younger than 65 years old: results after five to fifteen years. JShoulder Elbow Surg 22(9):1199-1208

6. Ernstbrunner L, Suter A, Catanzaro S, Rahm S, GerberC(2017) Reverse total shoulderarthroplasty for massive, irreparable rotator cuff tears before the age of 60 years: long-term results. J Bone Joint Surg Am 99(20):1721-1729

7. Gerber C, Canonica S, Catanzaro S, Ernstbrunner L (2018) Longitudinal observational study of reverse total shoulder arthroplasty for irreparable rotator cuff dysfunction: results after 15 years. J Shoulder Elbow Surg 27(5):831-838

8. Cuff DJ, Pupello DR, Santoni BG, Clark RE, Frankle MA (2017) Reverse shoulder arthroplasty for the treatment of rotator cuff deficiency: a concise follow-up, at a minimum of 10 years, of previous reports. J Bone Joint Surg Am 99(22):1895-1899

9. Bacle G, Nove-Josserand L, Garaud P, Walch G (2017) Long-term outcomes of reverse total shoulder arthroplasty: a follow-up of a previous study. JBone Joint Surg Am 99(6):454-461

10. Bassens D, Decock T, Van Tongel A, De Wilde $L$ (2019) Long-term results of the Delta Xtend reverse shoulder prosthesis. JShoulder Elbow Surg 28(6):1091-1097

11. Favard L, Levigne C, Nerot C, Gerber C, De Wilde L, Mole D (2011) Reverse prostheses in arthropathies with cuff tear: are survivorship and function maintained over time? Clin Orthop Relat Res 469(9):2469-2475

12. Brunet J, Berhouet J, Chelli M, Favard L (2016) Reverse prosthesis for cuff tear arthritis - (Hamada IV and V) non operated. In: Boileau P, Walch G, Molé D, Favard L, Lévigne C, Sirveaux F et al (Hrsg) Shoulder concepts: reverse shoulder arthroplasty. Sauramps Medical, Montpellier, S61-69

13. Cutlip RG, Baker BA, Geronilla KB, Mercer RR, Kashon ML, Miller GR et al (2006) Chronic exposure to stretch-shortening contractions results in skeletal muscle adaptation in young rats and maladaptation in old rats. Appl Physiol Nutr Metab 31(5):573-587

14. Baker BA, Hollander MS, Mercer RR, Kashon ML, Cutlip RG (2008) Adaptive stretch-shortening contractions: diminished regenerative capacity with aging. Appl Physiol Nutr Metab 33(6):1181-1191

15. Faulkner JA, Brooks SV, Zerba E (1995) Muscle atrophy and weakness with aging: contractioninduced injury as an underlying mechanism. J Gerontol A Biol Sci Med Sci 50:124-129

16. Kircher J (2012) Die Omarthrose: Einteilung, Morphologie und Diagnostik. Obere Extrem 7:91-99

17. Bercik MJ, Kruse K 2nd, Yalizis M, Gauci MO, Chaoui J, Walch G (2016) A modification to the Walch classification of the glenoid in primary glenohumeral osteoarthritis using three-dimensional imaging. J Shoulder Elbow Surg 25(10):1601-1606

18. Walch G, Badet R, Boulahia A, Khoury A (1999) Morphologic study of the glenoid in primary glenohumeral osteoarthritis. J Arthroplasty 14(6):756-760

19. Mizuno N, Denard PJ, Raiss P, Walch G (2013) Reverse total shoulder arthroplasty for primary glenohumeral osteoarthritis in patients with a biconcave glenoid. J Bone Joint Surg Am 95(14):1297-1304

20. Collin P, Herve A, Walch G, Boileau P, Muniandy M, Chelli M (2019) Mid-term results of reverse shoulder arthroplasty for glenohumeral osteoarthritis with posterior glenoid deficiency and humeral subluxation. J Shoulder Elbow Surg 28(10):2023-2030

21. Neer C (1990) Glenohumeral arthropathy in rheumatoid arthritis. In: Neer CS II (Hrsg) Shoulder reconstruction. Saunders, Philadelphia, S216-222

22. Woodruff MJ, Cohen AP, Bradley JG (2003) Arthroplasty of the shoulder in rheumatoid arthritis with rotator cuff dysfunction. Int Orthop 27(1):7-10

23. Levigne $C$ (2016) Reverse shoulder arthroplasty in rheumatoid arthritis. In: Boileau P, Walch G, Molé D, Favard L, Lévigne C, Sirveaux Fet al (Hrsg) Shoulder concepts: reverse shoulder arthroplasty. Sauramps Medical, Montpellier, S95-105
24. Palvanen M, Kannus P, Niemi S, Parkkari J (2006) Update in the epidemiology of proximal humeral fractures. Clin Orthop Relat Res 442:87-92

25. Roux A, Decroocq L, El Batti S, Bonnevialle N, Moineau G, Trojani C et al (2012) Epidemiology of proximal humerus fractures managed in a trauma center. Orthop Traumatol Surg Res 98(6):715-719

26. Sebastia-Forcada E, Cebrian-Gomez R, LizaurUtrilla A, Gil-Guillen V (2014) Reverse shoulder arthroplasty versus hemiarthroplasty for acute proximal humeral fractures. A blinded, randomized, controlled, prospective study. J Shoulder Elbow Surg 23(10):1419-1426

27. Cuff DJ, Pupello DR (2013) Comparison of hemiarthroplasty and reverse shoulder arthroplasty for the treatment of proximal humeral fractures in elderly patients. J Bone Joint Surg Am 95(22):2050-2055

28. Ferrel JR, Trinh TQ, Fischer RA (2015) Reverse total shoulder arthroplasty versus hemiarthroplasty for proximal humeral fractures: a systematic review. JOrthop Trauma 29(1):60-68

29. Peters PM, Plachel F, Danzinger V, Novi M, Mardian S, Scheibel M et al (2020) Clinical and radiographic outcomes after surgical treatment of proximal humeral fractures with head-split component. J Bone Joint Surg Am 102(1):68-75

30. Cazeneuve JF, Cristofari DJ (2011) Long term functional outcome following reverse shoulder arthroplasty in the elderly. Orthop Traumatol Surg Res 97(6):583-589

31. Decroocq L, Sirveaux F, Clavert P, Favard L, Boileau P (2016) Long-term results of RSA for acute fractures. In: Boileau P, Walch G, Molé D, Favard L, Lévigne C, Sirveaux F et al (Hrsg) Shoulde concepts: reverse shoulder arthroplasty. Sauramps Medical, Montpellier, S107-108

32. Boileau P, Walch G, Trojani C, Sinnerton R, Romeo A, Veneau B (1999) Sequelae of fractures of the proximal humerus: surgical classification and limits of shoulder arthroplasty. In: Walch G, Boileau P (Hrsg) Shoulder arthroplasty. Springer, Berlin, S349-358

33. Seeto B, Clowez G, Neyton L, Trojani C, Boileau P (2016) Reverse shoulder arthroplasty for fracture sequelae:a minimum 5 yearfollow-up. In:Boileau $P_{\text {, }}$ Walch G, Molé D, Favard L, Lévigne C, Sirveaux F et al (Hrsg) Shoulder concepts: reverse shoulder arthroplasty. Sauramps Medical, Montpellier, S 109-120

34. De Wilde L, Boileau P, Van der Bracht H (2011) Does reverse shoulder arthroplasty for tumors of the proximal humerus reduce impairment? Clin Orthop Relat Res 469(9):2489-2495

35. Sirveaux F, Jullion S, Raynier J-L (2016) Reverse prothesis for proximal humeral tumor. In: Boileau $P$, Walch G, Molé D, Favard L, Lévigne C, Sirveaux F et al (Hrsg) Reverse shoulder arthroplasty for nonoperated, irreperable massive cuff tear (Hamada I-II-III) minimum 5-year follow up. Sauramps Medical, Montpellier, S 121-132

36. D'Ollonne T, Baring T, Gendre T, Cavalier M, Chelli M, Sabbah Y et al (2016) Reverse shoulder arthroplasty for revision of failed shoulder hemiarthroplasty-Results at a minimum of 5 years follow-up. In: Boileau $P$, Walch G, Molé $D$, Favard L, Lévigne C, Sirveaux Fet al (Hrsg) Shoulder concepts: reverse shoulder arthroplasty. Sauramps Medical, Montpellier, S133-140

37. Gauci $M O$, Barret $H$, Cavalier $M$, Bessière $C$, Baring T, Favard L et al (2016) Long term results of reverse shoulder arthroplasty for revision after failed anatomical total shoulder arthtoplasty. In: Boileau P, Walch G, Molé D, Favard L, Lévigne C, 
Sirveaux $\mathrm{F}$ et al (Hrsg) Shoulder concepts:

reverse shoulder arthroplasty. Sauramps Medical, Montpellier, S141-145

38. Beck S, Patsalis T, Busch A, Dittrich F, Dudda M, Jager $M$ et al (2019) Long-term results of the reverse Total Evolutive Shoulder System (TESS). Arch Orthop Trauma Surg 139(8):1039-1044

39. Teissier $P$, Teissier J (2016) The TESS RSA for cuff deficient shoulders. In: Boileau P, Walch G, Molé D, Favard L, Lévigne C, Sirveaux F etal (Hrsg) Shoulder concepts: reverse shoulder arthroplasty. Sauramps Medical, Montpellier, S165-172

40. Ascione F, Domos P, Guarrella V, Chelli M, Boileau P, Walch G (2018) Long-term humeral complications after Grammont-style reverse shoulder arthroplasty. J Shoulder Elbow Surg 27(6):1065-1071

41. van Ochten JHM, van der Pluijm M, Pouw M, Felsch QTM, Heesterbeek P, de Vos MJ (2019) Long - term survivorship and clinical and radiological follow-up of the primary uncemented Delta III reverse shoulder prosthesis. J Orthop 16(4):342-346 